\title{
On the Action Formalism of Time-Dependent Density-Functional Theory
}

\author{
Martín A. Mosquera \\ Department of Chemistry \\ Purdue University \\ West Lafayette, IN 47907, USA \\ mmosquer@purdue .edu
}

May 21, 2013

\begin{abstract}
The Runge-Gross [E. Runge, and E. K. U. Gross, Phys. Rev. Lett., 52, 997 (1984)] action functional of time-dependent density-functional theory leads to a well-known causality paradox, i.e., a perturbation of the electronic density in the future affects the response of the system in the present. This paradox is known to be caused by an inconsistent application of the Dirac-Frenkel variational principle. In view of the recent solutions to this problem, the action functional employed by Runge and Gross in their formulation of time-dependent density functional theory is analyzed in the context of the Keldysh contour technique. The time-dependent electronic density, as well as the concept of causality, are extended to the contour. We derive a variational equation that obeys causality and relates the exchange-correlation potential with its kernel, and the functional derivative of the exchange-correlation action functional with respect to the density. It is shown that the adiabatic local-density approximation is a consistent solution of this equation and that the time-dependent optimized potential method can also be derived from it. The formalism presented here can be used to find new approximations methods to the exchange-correlation potential and avoid the causality dilemma.
\end{abstract}

\section{Introduction}

Time-dependent density-functional theory (TDDFT) [1-3] establishes the time-dependent (TD) electronic density as the primary object of study to understand the dynamics of molecular systems. TDDFT is widely used to calculate spectroscopic properties of molecules and solids, specially when TD perturbation theory is not applicable [4]. However, TDDFT can also be used to study electronic excitations in the linear regime, or predict the electronic 
ground-state energy and density $[5,6]$. The foundation of TDDFT is the theorem of Runge and Gross (RG) [7] stating that there is a one-to-one mapping, given an initial state, between electronic TD densities and TD external potentials. Later van Leeuwen [8] showed that it is possible to reproduce the TD electronic density of the system of interacting electrons by a system of non-interacting electrons, which makes possible the use of the TD Kohn-Sham (KS) equations. Challenges in TDDFT include the correct description of charge transfer excitation $[9,10]$, electronic transport through a molecule connected to metallic leads under a bias [11-13], high-order harmonic generation [14], double excitations [15], van der Waals interactions, among others [16].

In TDDFT, the prediction of the evolution of the electronic density is reformulated in terms of the TD KS equations, which are easier to solve than the time-dependent Schrödinger equation (TDSE). Moreover, every observable of the system can be expressed as a functional of the density because the wave function is a density-functional as well. However, Runge and Gross [7] proved that the one-to-one mapping between TD densities and TD potentials is valid under the restriction that the TD external potential is Taylor-expandable in terms of the time variable. The question as to how vast the set of TD potentials (or TD densities) of the RG theorem should be is still an open question. The proof shown by Ruggenthaler and van Leeuwen [17] and Ruggenthaler et al. [18] suggests that the Runge-Gross theorem can be extended to a wider set of TD potentials including those that are non-analytic in time. Despite it is known that this map exists and there is a formal procedure to construct KS potentials, it is still a challenge to calculate the TD potential from a given TD density [18-20].

In ground-state density-functional theory (DFT), the exchange-correlation (XC) potential is expressed as the functional derivative of the $\mathrm{XC}$ energy functional with respect to the time-independent electronic density. In TDDFT, an analogous variational relation between the TD XC potential and its action functional has been sought for the last three decades. Peuckert [21] first suggested that the Dirac-Frenkel action functional and its variational principle should be used in TDDFT. Later Runge and Gross [7] showed that the DiracFrenkel action functional extended to TDDFT leads to identify the TD XC potential as a functional derivative of the $\mathrm{XC}$ action functional with respect to the density. However, it was later found by Gross et al. [22] that this gives rise to a paradox in which a variation of the density in the future induces a perturbation of the potential in the past. According to this, the inverse first order response functional would not be causal. Gross et al. [22] conjectured that the paradox could be solved by incorporating the causality principle explicitly into the action formalism.

To resolve this causality paradox several works have been published. Rajagopal [23] introduced an action based on the work of Jackiw and Kerman [24] in quantum field theory. However, this formalism does not use the density as basic variable but a transition density that can be negative-valued; this quantity is unsuitable as a basic quantity for TDDFT.

van Leeuwen [25] proposed a functional in the Keldysh contour with similar properties to that of a free energy. This functional depends on a pseudo-density in the Keldysh space that reduces to the density of the system when the potential in the Keldysh space corresponds 
to a physical potential. Due to the symmetry properties of the first-order response function of the pseudo-density, the causality is restored when the density is mapped to the real-time regime. However, the van Leeuwen formalism requires expansion of the action functional in terms of Feynman diagrams, while the functional of RG does not require such expansion. Furthermore, the operator used in this formalism for the pseudo-density is not Hermitian in general and thus the pseudo-density does not integrate to the total number of electrons of the system; except when the density is physical.

Recently, Vignale [26] solved the causality paradox in real-time by showing that the source of the problem in the RG formulation is a boundary condition. He showed that only the initial condition is necessary in the Runge-Gross functional to recover the causality restriction in general, and derived an expression for the $\mathrm{XC}$ potential that is causal.

In this paper I review Vignale's solution of the causality paradox in real-time from the perspective of unitary propagation and later use Vignale's theory to extend the RG action functional to the Keldysh contour. The RG action functional in the Keldysh space, unlike the van Leeuwen functional, does not require diagrammatic expansion and uses an electronic density that is a causal functional of the potential in the Keldysh space. By the RG theorem applied to the Keldysh space and under the assumption that the density is a strictly causal functional of the potential in the contour, I show that a variational equation relating the $\mathrm{XC}$ potential with the $\mathrm{XC}$ action functional arises. This equation shows an explicit dependence on the memory of the system through the XC kernel. I show that the adiabatic local density approximation (ALDA) is consistent with this equation, how the TD optimized potential method (TDOPM) can be obtained, and also how the ground-state $\mathrm{XC}$ potential can be recovered.

\section{Real-time Analysis}

The Dirac-Frenkel variational principle provides a method to derive the TDSE and its approximations by finding a stationary value of the action functional:

$$
W[\psi ; v]=\int_{t_{0}}^{t_{1}} \mathrm{~d} t\left\langle\psi(t)\left|\mathrm{i} \frac{\partial}{\partial t}-\hat{H}[v](t)\right| \psi(t)\right\rangle .
$$

In this work we consider Hamiltonians of the form:

$$
\hat{H}[v](t)=\hat{T}+\hat{W}+\int \mathrm{d}^{3} \mathbf{r} v(\mathbf{r} t) \hat{n}(\mathbf{r})
$$

where $\hat{T}$ and $\hat{W}$ are the kinetic energy and electron-electron repulsion energy operators, respectively, and $\hat{n}(\mathbf{r})$ is the density operator. The Dirac-Frenkel functional is defined over a Hilbert space of antisymmetric wavefunctions representing bound systems of $N$ electrons. The TD Schrödinger equation (TDSE) is thus obtained by setting

$$
\delta_{\psi} W[\psi ; v]=0
$$


This variational principle supposes that $\delta \psi\left(t_{0}\right)=\delta \psi\left(t_{1}\right)=0$. The solution of this equation, denoted as $\psi[v](t)$, is the solution of the TDSE:

$$
\mathrm{i} \frac{\partial}{\partial t}|\psi[v](t)\rangle=\hat{H}[v](t)|\psi[v](t)\rangle
$$

$|\psi[v](t)\rangle$ is said to be a $v$-representable ket in real-time, which expressed in terms of the unitary evolution operator is:

$$
|\psi[v](t)\rangle=\hat{U}[v]\left(t, t_{0}\right)\left|\psi\left(t_{0}\right)\right\rangle
$$

where

$$
\hat{U}[v]\left(t, t_{0}\right)=\hat{\mathcal{T}} \exp \left(-\mathrm{i} \int_{t_{0}}^{t} \mathrm{~d} s \hat{H}[v](s)\right) .
$$

Here $\hat{\mathcal{T}}$ is the time-ordering operator in real-time. In this work we interpret the integral in the above equation to be taken over the interval $\left[t_{0}, t\right)$, i.e.

$$
\int_{t_{0}}^{t} \mathrm{~d} s \hat{H}[v](s) \mathrm{d} s=\lim _{\epsilon \rightarrow 0} \int_{t_{0}}^{t-|\epsilon|} \mathrm{d} s \hat{H}[v](s) .
$$

Note that the ket $|\psi[v](t)\rangle$ is a causal functional of the potential: It is determined by the potential $v$ at times less than $t$. We refer to this dependency on the potential $(\mathrm{Eq}(6))$ as the strict causality assumption.

All observables of $\psi$ are also causal functionals of $u$. For example, the density of the system,

$$
n[v](\mathbf{r} t)=\left\langle\psi\left(t_{0}\right)\left|\hat{U}^{\dagger}[v]\left(t, t_{0}\right) \hat{n}(\mathbf{r}) \hat{U}[v]\left(t, t_{0}\right)\right| \psi\left(t_{0}\right)\right\rangle,
$$

is determined by $v$ in the interval $\left[t_{0}, t\right)[3]$. By the $\mathrm{RG}$ theorem, given a fixed initial state, the potential $v$ at times in $\left[t_{0}, t\right)$ uniquely determines $n$ in the interval $\left[t_{0}, t\right)$, and vice versa. If we denote as $u[n]$ the external potential as a functional of the TD density, then a first order variation in $u$ is given by a variation of $n$ over the interval $\left[t_{0}, t\right)$ :

$$
\delta u[n](\mathbf{r} t)=\int_{t_{0}}^{t} \mathrm{~d} t^{\prime} \int \mathrm{d}^{3} \mathbf{r}^{\prime} \chi^{-1}[n]\left(\mathbf{r} t, \mathbf{r}^{\prime} t^{\prime}\right) \delta n\left(\mathbf{r}^{\prime} t^{\prime}\right)
$$

where

$$
\chi^{-1}[n]\left(\mathbf{r} t, \mathbf{r}^{\prime} t^{\prime}\right)=\frac{\delta u(\mathbf{r} t)}{\delta n\left(\mathbf{r}^{\prime} t^{\prime}\right)} .
$$

This indicates that $\delta u(\mathbf{r} t) / \delta n\left(\mathbf{r}^{\prime} t^{\prime}\right)$ for $t \leq t^{\prime}$ is not defined because it does not contribute to the integral of Eq. (9). However, for convenience we set:

$$
\chi^{-1}[n]\left(\mathbf{r} t, \mathbf{r}^{\prime} t^{\prime}\right)=0 \quad t \leq t^{\prime} .
$$

Vignale [26], however, employing the evolution equation of the current, showed that $\delta u(\mathbf{r} t) / \delta n\left(\mathbf{r}^{\prime} t^{\prime}\right)$ is related to $\delta\left(t-t^{\prime}\right)$ and its first and second order time-derivatives when 
$t=t^{\prime}$. This result is obtained under two assumptions different from ours: First, $u(\mathbf{r} t)$ is determined by $n\left(\mathbf{r}^{\prime} t^{\prime}\right)$ for $t^{\prime} \leq t$. And secondly, the functional derivative of the stress tensor with respect to the density vanishes at equal times. Our assumption avoids this singularity in $\chi^{-1}$ and will be used to simplify our calculations in the Keldysh space (section 3).

Now let us consider the Runge-Gross action functional:

$$
A_{v}[n]=\int_{t_{0}}^{t_{1}} \mathrm{~d} t\left\langle\psi[n](t)\left|\mathrm{i} \frac{\partial}{\partial t}-\hat{H}[v](t)\right| \psi[n](t)\right\rangle
$$

where $|\psi[n](t)\rangle=|\psi[u[n]](t)\rangle, v$ is some TD external potential, and $t_{1}>t_{0}$. Note that the ket $|\psi[n](t)\rangle$ is causal, i.e., it is determined by $n$ in the interval $\left[t_{0}, t\right)$. Runge and Gross [7], based on the Dirac-Frenkel variational principle, imposed $\delta \psi\left(t_{0}\right)=\delta \psi\left(t_{1}\right)=0$ and

$$
\frac{\delta A_{v}}{\delta n(\mathbf{r} t)}=0
$$

which leads to the following alternative form of the variational principle:

$$
\frac{\delta B[n]}{\delta n(\mathbf{r} t)}-v(\mathbf{r} t)=0
$$

where $B[n]$ is the internal action:

$$
B[n]=\int_{t_{0}}^{t_{1}} \mathrm{~d} t\left\langle\psi[n](t)\left|\mathrm{i} \frac{\partial}{\partial t}-\hat{T}-\hat{W}\right| \psi[n](t)\right\rangle .
$$

If Eq. (14) were valid then we could assert that [27]:

$$
u[n](\mathbf{r} t)=\frac{\delta B[n]}{\delta n(\mathbf{r} t)}
$$

Unfortunately, when the above function is further differentiate with respect to $n$,

$$
\chi\left(\mathbf{r} t, \mathbf{r}^{\prime} t^{\prime}\right)=\frac{\delta^{2} B[n]}{\delta n\left(\mathbf{r}^{\prime} t^{\prime}\right) \delta n(\mathbf{r} t)},
$$

one finds an inconsistency because the above equation implies that $\chi\left(\mathbf{r} t, \mathbf{r}^{\prime} t^{\prime}\right) \neq 0$ for $t<t^{\prime}$. This is known as the causality paradox [22]. The solution to the paradox was found by Vignale [26], who pointed out that, according to the definition of $v$-representable wavefunction, we can only set $\delta \psi\left(t_{0}\right)=0$ because a perturbation $\delta n(\mathbf{r} t)$, in general, will induce a response $\delta \psi\left(t_{1}\right) \neq 0$.

The solution of Vignale [26] can be viewed as a direct implementation of the causality principle into the RG functional. For example, the internal action $B[n]$, using the TDSE, can be written as [3]:

$$
B[n]=\int_{t_{0}}^{t_{1}} \mathrm{~d} t \int \mathrm{d}^{3} \mathbf{r} u[n](\mathbf{r} t) n(\mathbf{r} t)
$$


The density-functional $u[n]$ is causal by the $R G$ theorem. If we differentiate the above functional with respect to the density and insert the result into the functional derivative of the RG action functional we obtain

$$
\frac{\delta A_{v}}{\delta n(\mathbf{r} t)}=u[n](\mathbf{r} t)-v(\mathbf{r} t)+\int_{t}^{t_{1}} \mathrm{~d} t^{\prime} \int \mathrm{d}^{3} \mathbf{r}^{\prime} \chi^{-1}[n]\left(\mathbf{r} t, \mathbf{r}^{\prime} t^{\prime}\right) n\left(\mathbf{r}^{\prime} t^{\prime}\right) .
$$

Now let $n_{v}$ be the TD density corresponding to $v$, then:

$$
\left.\frac{\delta A_{v}}{\delta n(\mathbf{r} t)}\right|_{n=n_{v}}=\int_{t}^{t_{1}} \mathrm{~d} t^{\prime} \int \mathrm{d}^{3} \mathbf{r}^{\prime} \chi^{-1}\left[n_{v}\right]\left(\mathbf{r} t, \mathbf{r}^{\prime} t^{\prime}\right) n_{v}\left(\mathbf{r}^{\prime} t^{\prime}\right) .
$$

This last equation is an alternative form of the Vignale variational formulation that shows that $n_{v}$ is not a stationary value of $A_{v}[n]$. This is a consequence of constraining the wavefunctions of the $\mathrm{RG}$ functional to be density-functionals of the form $\psi[u[n]]$. Ruggenthaler and Leeuwen [27] showed that not every TD wave-function can be associated with a TD external potential (or a TD density). Hence the domain of the RG functional is just a subset of the domain of the Dirac-Frenkel functional, explaining why the RG and the Dirac-Frenkel functionals lead to different results.

\section{Keldysh-space Analysis}

Let us add a super index + or - to the time variable $t$. The Keldysh contour, $\mathcal{C}$, is expressed as $\mathcal{C}=\mathcal{C}^{+} \cup \mathcal{C}^{-}$, where $\mathcal{C}^{+}=\left[t_{0}^{+}, t_{1}^{+}\right]$and $\mathcal{C}^{-}=\left[t_{0}^{-}, t_{1}^{-}\right]$. We denote $z$ as a variable in the contour $\mathcal{C}$, and let $z_{\mathrm{i}}=t_{0}^{+}$and $z_{\mathrm{f}}=t_{1}^{-}$. The arrow of time in $\mathcal{C}$ points from $t_{0}^{+}$to $t_{1}^{+}$and from $t_{1}^{-}$to $t_{0}^{-}$. Thus, any $z \in \mathcal{C}^{-}$is said to be later than any $z^{\prime} \in \mathcal{C}^{+}$. If $z, z^{\prime} \in \mathcal{C}^{-}$we say that $z$ is later than $z^{\prime}$ if $t(z)<t\left(z^{\prime}\right)$, where $t(z)$ is the real value of $z$. A ket in $\mathcal{C}$ is denoted as $\left|\psi_{\mathrm{c}}\left[u_{\mathrm{c}}\right](z)\right\rangle$, where $u_{\mathrm{c}}(\mathbf{r} z)$ is some potential in $\mathcal{C}$. A physical potential in $\mathcal{C}$ is denoted as $\bar{u}_{\mathrm{c}}$ and it satisfies $\bar{u}_{\mathrm{c}}\left(\mathbf{r} t^{+}\right)=\bar{u}_{\mathrm{c}}\left(\mathbf{r} t^{-}\right)$. Thus a potential in real-time is mapped to the Keldysh space when setting $\bar{u}_{\mathrm{c}}\left(\mathbf{r} t^{ \pm}\right)=u(\mathbf{r} t)\left(t^{ \pm}\right.$we denotes evaluation at $\mathcal{C}^{+}$or $\left.\mathcal{C}^{-}\right)$

We now extend the unitary propagator $\hat{U}$ to the Keldysh space as follows:

$$
\hat{U}_{\mathrm{c}}\left[u_{\mathrm{c}}\right]\left(z, z_{\mathrm{i}}\right)=\hat{\mathcal{T}}_{\mathcal{C}} \exp \left[-\mathrm{i} \int_{z_{\mathrm{i}}}^{z} \mathrm{~d} z^{\prime} \hat{H}_{\mathrm{c}}\left[u_{\mathrm{c}}\right]\left(z^{\prime}\right)\right]
$$

where $\hat{\mathcal{T}}_{\mathcal{C}}$ is the path-ordering operator in $\mathcal{C}$ (for example, $\hat{\mathcal{T}}_{\mathcal{C}}\left[\hat{B}_{\mathrm{c}}\left(z^{\prime}\right) \hat{A}_{\mathrm{c}}(z)\right]=\hat{A}_{\mathrm{c}}(z) \hat{B}_{\mathrm{c}}\left(z^{\prime}\right)$ if $z$ is later than $\left.z^{\prime}\right)$. The Hamiltonian in the Keldysh space now reads $\hat{H}_{\mathrm{c}}\left[u_{\mathrm{c}}\right](z)=\hat{T}+\hat{W}+$ $\int \mathrm{d}^{3} \mathbf{r} u_{\mathrm{c}}(\mathbf{r} z) \hat{n}(\mathbf{r})$. The integration over the pseudo-time is defined as:

$$
\int_{z_{\mathrm{i}}}^{z} \mathrm{~d} z^{\prime} f_{\mathrm{c}}\left(z^{\prime}\right)=\left\{\begin{array}{l}
\int_{t_{0}}^{t} \mathrm{~d} t^{\prime} f_{\mathrm{c}}\left(t^{\prime+}\right), \quad z=t^{+} \\
\int_{t_{0}}^{t_{1}} \mathrm{~d} t^{\prime} f_{\mathrm{c}}\left(t^{\prime+}\right)+\int_{t_{1}}^{t} \mathrm{~d} t^{\prime} f_{\mathrm{c}}\left(t^{\prime-}\right), \quad z=t^{-}
\end{array}\right.
$$

A $v$-representable ket in $\mathcal{C}$ is thus expressed as $\left|\psi_{\mathrm{c}}\left[u_{\mathrm{c}}\right](z)\right\rangle=\hat{U}_{\mathrm{c}}\left[u_{\mathrm{c}}\right]\left(z, z_{\mathrm{i}}\right)\left|\psi_{\mathrm{c}}\left(z_{\mathrm{i}}\right)\right\rangle$, where $\left|\psi_{\mathrm{c}}\left(z_{\mathrm{i}}\right)\right\rangle=\left|\psi\left(t_{0}\right)\right\rangle$ is the initial state of the system. Note that $\psi_{\mathrm{c}}(z)$ does not depend on the 
potential $u_{\mathrm{c}}$ at later times than $z$. As in the real-time case, we assume that the end point of the integral in Eq. (21) is not included; this can be considered as an extension of the strict causality assumption to the Keldysh contour. We define the density in $\mathcal{C}$ as [28]:

$$
n_{\mathrm{c}}\left[u_{\mathrm{c}}\right](\mathbf{r} z)=\left\langle\hat{U}_{\mathrm{c}}^{\dagger}\left[u_{\mathrm{c}}\right]\left(z, z_{\mathrm{i}}\right) \hat{n}(\mathbf{r}) \hat{U}\left[u_{\mathrm{c}}\right]_{\mathrm{c}}\left(z, z_{\mathrm{i}}\right)\right\rangle
$$

where $\langle\cdot\rangle=\left\langle\psi_{\mathrm{c}}\left(z_{\mathrm{i}}\right)|\cdot| \psi_{\mathrm{c}}\left(z_{\mathrm{i}}\right)\right\rangle$. To prove that there is a one-to-one mapping between $n_{\mathrm{c}}$ and $u_{\mathrm{c}}$, it is sufficient to notice that $\psi_{\mathrm{c}}$ satisfies the Schrödinger equation in $\mathcal{C}^{+}$. Therefore, if the potential can be expressed as a power series around $z_{\mathrm{i}}$, then the RG theorem and its extension [17] including non-analytic potentials apply in this case.

Let us examine the action functional proposed by van Leeuwen [25], which reads

$$
A_{\mathrm{vL}}\left[u_{\mathrm{c}}\right]=\mathrm{i} \ln \left\langle\hat{U}_{\mathrm{c}}\left[u_{\mathrm{c}}\right]\left(z_{\mathrm{f}}, z_{\mathrm{i}}\right)\right\rangle .
$$

The functional derivative of this functional with respect to the potential $u_{\mathrm{c}}$ yields the pseudodensity [25]:

$$
n_{\mathrm{vL}}(\mathbf{r} z)=\frac{\left\langle\hat{U}_{\mathrm{c}}\left(z_{\mathrm{f}}, z\right) \hat{n}(\mathbf{r}) \hat{U}_{\mathrm{c}}\left(z, z_{\mathrm{i}}\right)\right\rangle}{\left\langle\hat{U}_{\mathrm{c}}\left(z_{\mathrm{f}}, z_{\mathrm{i}}\right)\right\rangle} .
$$

However, the above density is an average of the operator:

$$
\hat{n}_{\mathrm{vL}, \mathrm{H}}(\mathbf{r})=\hat{U}_{\mathrm{c}}\left(z_{\mathrm{f}}, z\right) \hat{n}(\mathbf{r}) \hat{U}_{\mathrm{c}}\left(z, z_{\mathrm{i}}\right)
$$

which is not a Hermitian operator. Therefore

$$
\int \mathrm{d}^{3} \mathbf{r} \hat{n}_{\mathrm{vL}, \mathrm{H}}(\mathbf{r} z)=\hat{N} \hat{U}_{\mathrm{c}}\left(z_{\mathrm{f}}, z_{\mathrm{i}}\right)
$$

where $\hat{N}$ is the particle-number operator. This implies that $n_{\mathrm{vL}}$ does not integrate to $N$; except when the potential $u_{\mathrm{c}}$ is physical [25]. The density $n_{\mathrm{c}}$, on the other hand, integrates to $N$ and is always positive.

It can be shown that the response function of the density in $\mathcal{C}$ is given by:

$$
\chi_{\mathrm{c}}\left[u_{\mathrm{c}}\right]\left(\mathbf{r} z, \mathbf{r}^{\prime} z^{\prime}\right)=\frac{\delta n_{\mathrm{c}}(\mathbf{r} z)}{\delta u_{\mathrm{c}}\left(\mathbf{r}^{\prime} z^{\prime}\right)}=-\mathrm{i}\left\langle\left[\hat{n}_{\mathrm{c}, \mathrm{H}}\left[u_{\mathrm{c}}\right](\mathbf{r} z), \hat{n}_{\mathrm{c}, \mathrm{H}}\left[u_{\mathrm{c}}\right]\left(\mathbf{r}^{\prime} z^{\prime}\right)\right]\right\rangle
$$

where the Heisenberg representation of the density operator $\hat{n}(\mathbf{r})$ is

$$
\hat{n}_{\mathrm{c}, \mathrm{H}}\left[u_{\mathrm{c}}\right](\mathbf{r} z)=\hat{U}_{\mathrm{c}}^{\dagger}\left[u_{\mathrm{c}}\right]\left(z, z_{\mathrm{i}}\right) \hat{n}(\mathbf{r}) \hat{U}_{\mathrm{c}}\left[u_{\mathrm{c}}\right]\left(z, z_{\mathrm{i}}\right) .
$$

Eq. (28) is valid if $z$ is later than $z^{\prime}$, and we set $\chi_{\mathrm{c}}\left(\mathbf{r} z, \mathbf{r}^{\prime} z^{\prime}\right)=0$ if $z^{\prime}$ is later than or equal to $z$.

The inverse first order response function $\chi_{\mathrm{c}}^{-1}\left[n_{\mathrm{c}}\right]\left(\mathbf{r} z, \mathbf{r}^{\prime} z^{\prime}\right)=\delta u_{\mathrm{c}}(\mathbf{r} z) / \delta n_{\mathrm{c}}\left(\mathbf{r}^{\prime} z^{\prime}\right)$, according to the RG theorem extended to the $\mathcal{C}$, must also satisfy causality in the contour, e.g., $\chi_{\mathrm{c}}^{-1}\left(\mathbf{r} z, \mathbf{r} z^{\prime}\right)=0$ if $z=z^{\prime}$ or $z^{\prime}$ is later than $z$. When a physical potential is used, the Heinsenberg operators recover their usual form in real-time. Therefore, we obtain a physical 
density $\bar{n}_{\mathrm{c}}\left(\mathbf{r} t^{ \pm}\right)=n(\mathbf{r} t)$. From Eq. (28) we can show that the first order response function satisfies the antisymmetry relationship:

$$
\left.\chi_{\mathrm{c}}\left(\mathbf{r} t^{+}, \mathbf{r}^{\prime} t^{\prime+}\right)\right|_{u_{\mathrm{c}}=\bar{u}_{\mathrm{c}}}=-\left.\chi_{\mathrm{c}}\left(\mathbf{r}^{\prime} t^{\prime-}, \mathbf{r} t^{-}\right)\right|_{u_{\mathrm{c}}=\bar{u}_{\mathrm{c}}}
$$

where $u_{\mathrm{c}}=\bar{u}_{\mathrm{c}}$ denotes evaluation at the physical regime. Note that $\chi_{\mathrm{c}}$ also satisfies $\chi_{\mathrm{c}}\left(\mathbf{r} t^{+}, \mathbf{r}^{\prime} t^{\prime+}\right)=\chi_{\mathrm{c}}\left(\mathbf{r} t^{-}, \mathbf{r}^{\prime} t^{\prime+}\right)$ and $\chi_{\mathrm{c}}\left(\mathbf{r}^{\prime} t^{\prime-}, \mathbf{r} t^{+}\right)=\chi_{\mathrm{c}}\left(\mathbf{r}^{\prime} t^{\prime-}, \mathbf{r} t^{-}\right)$if $t>t^{\prime}$ and $u_{\mathrm{c}}=\bar{u}_{\mathrm{c}}$.

The response of the density in the Keldysh space is [25]:

$$
\delta n_{\mathrm{c}}\left[u_{\mathrm{c}}\right](\mathbf{r} z)=\int_{z_{\mathrm{i}}}^{z_{\mathrm{f}}} \mathrm{d} z^{\prime} \int \mathrm{d}^{3} \mathbf{r}^{\prime} \chi_{\mathrm{c}}\left[u_{\mathrm{c}}\right]\left(\mathbf{r} z, \mathbf{r}^{\prime} z^{\prime}\right) \delta u_{\mathrm{c}}\left(\mathbf{r}^{\prime} z^{\prime}\right) .
$$

To obtain the response in real-time, the variation of a physical potential must satisfy $\delta \bar{u}_{\mathrm{c}}\left(\mathbf{r} t^{+}\right)=\delta \bar{u}_{\mathrm{c}}\left(\mathbf{r} t^{-}\right)=\delta u(\mathbf{r} t)$. Using the aforementioned properties of $\chi_{\mathrm{c}}$ to calculate the above integral, the response of the density turns out to be independent of the time location in the contour, i.e. $\delta n_{\mathrm{c}}\left(\mathbf{r} t^{+}\right)=\delta n_{\mathrm{c}}\left(\mathbf{r} t^{-}\right)=\delta n(\mathbf{r} t)$. Hence, it is determined by:

$$
\delta n_{\mathrm{c}}\left[\bar{u}_{\mathrm{c}}\right](\mathbf{r} t)=\int_{t_{0}}^{t^{ \pm}} \mathrm{d} t^{\prime} \int \mathrm{d}^{3} \mathbf{r}^{\prime} \chi_{\mathrm{c}}\left[\bar{u}_{\mathrm{c}}\right]\left(\mathbf{r} t^{ \pm}, \mathbf{r}^{\prime} t^{\prime+}\right) \delta \bar{u}_{\mathrm{c}}\left(\mathbf{r}^{\prime} t^{\prime+}\right) .
$$

This result allows us to identify the response in real-time $\chi\left(\mathbf{r} t, \mathbf{r}^{\prime} t^{\prime}\right)$ as $\left.\chi_{\mathrm{c}}\left(\mathbf{r} t^{ \pm}, \mathbf{r}^{\prime} t^{\prime+}\right)\right|_{u_{\mathrm{c}}=\bar{u}_{\mathrm{c}}}$ or $-\left.\chi_{\mathrm{c}}\left(\mathbf{r}^{\prime} t^{\prime-}, \mathbf{r} t^{-}\right)\right|_{u_{\mathrm{c}}=\bar{u}_{\mathrm{c}}}$, which are causal. Exchanging variables in the integral of $\chi_{\mathrm{c}} \chi_{\mathrm{c}}^{-1}$ reveals that $\chi_{\mathrm{c}}^{-1}$ satisfies the same relationships of $\chi_{\mathrm{c}}$ regarding exchange of variables at physical densities.

Let us extend the functional $A_{v}$ to the Keldysh space:

$$
\mathcal{A}_{\bar{v}_{\mathrm{c}}}\left[n_{\mathrm{c}}\right]=\mathcal{B}\left[n_{\mathrm{c}}\right]-\int_{z_{\mathrm{i}}}^{z_{\mathrm{f}}} \mathrm{d} z \int \mathrm{d}^{3} \mathbf{r} n_{\mathrm{c}}(\mathbf{r} z) \bar{v}_{\mathrm{c}}(\mathbf{r} z)
$$

where

$$
\mathcal{B}\left[n_{\mathrm{c}}\right]=\int_{z_{\mathrm{i}}}^{z_{\mathrm{f}}} \mathrm{d} z\left\langle\psi_{\mathrm{c}}\left[n_{\mathrm{c}}\right](z)\left|\mathrm{i} \frac{\partial}{\partial z}-\hat{T}-\hat{W}\right| \psi_{\mathrm{c}}\left[n_{\mathrm{c}}\right](z)\right\rangle,
$$

$\bar{v}_{\mathrm{c}}$ is some external physical potential, and $\partial f(z) / \partial z=\partial f\left(t^{\sigma}\right) / \partial t$, where $\sigma=+,-$. Vignale equation in this case reads:

$$
\begin{aligned}
\left.\frac{\delta \mathcal{B}}{\delta n_{\mathrm{c}}(\mathbf{r} z)}\right|_{n_{\mathrm{c}}=\bar{n}_{\mathrm{c}, \bar{v}_{\mathrm{c}}}} & -\bar{v}_{\mathrm{c}}(\mathbf{r} z)=\left.\mathrm{i}\left\langle\psi_{\mathrm{c}}\left(z_{\mathrm{f}}\right) \mid \frac{\delta \psi_{\mathrm{c}}\left(z_{\mathrm{f}}\right)}{\delta n_{\mathrm{c}}(\mathbf{r} z)}\right\rangle\right|_{n_{\mathrm{c}}=\bar{n}_{\mathrm{c}, \bar{v}_{\mathrm{c}}}} \\
& =\int_{z}^{z_{\mathrm{f}}} \mathrm{d} z^{\prime} \int \mathrm{d}^{3} \mathbf{r}^{\prime} \bar{n}_{\mathrm{c}, \overline{\mathrm{c}}_{\mathrm{c}}}\left(\mathbf{r}^{\prime} z^{\prime}\right) \chi_{\mathrm{c}}^{-1}\left[\bar{n}_{\mathrm{c}, \bar{v}_{\mathrm{c}}}\right]\left(\mathbf{r}^{\prime} z^{\prime}, \mathbf{r} z\right)
\end{aligned}
$$

The left hand side of the above equation corresponds to $\delta \mathcal{A}_{\bar{v}_{\mathrm{c}}} / \delta n_{\mathrm{c}}(\mathbf{r} z)$ evaluated at the density that yields $\bar{v}_{\mathrm{c}}, \bar{n}_{\mathrm{c}, \bar{v}_{\mathrm{c}}}$. Additionally, the above equation also gives the functional derivative $\delta \mathcal{B} / \delta n_{\mathrm{c}}(\mathbf{r} z)$ for an arbitrary density $n_{\mathrm{c}}$; in this case, we replace $\bar{v}_{\mathrm{c}}$ by $u_{\mathrm{c}}\left[n_{\mathrm{c}}\right](\mathbf{r} z), \bar{n}_{\mathrm{c}, \bar{v}_{\mathrm{c}}}$ by $n_{\mathrm{c}}$ and the inverse response function has to be evaluated at $n_{\mathrm{c}}$. 
Let us introduce the KS action functional:

$$
\mathcal{A}_{\mathrm{s}, \bar{v}_{c, s}}\left[n_{\mathrm{c}}\right]=\mathcal{B}_{\mathrm{s}}\left[n_{\mathrm{c}}\right]-\int_{z_{\mathrm{i}}}^{z_{\mathrm{f}}} \mathrm{d} z \int \mathrm{d}^{3} \mathbf{r} n_{\mathrm{c}}(\mathbf{r} z) \bar{v}_{\mathrm{c}, \mathrm{s}}(\mathbf{r} z),
$$

where $\bar{v}_{\mathrm{c}, \mathrm{s}}(\mathbf{r} z)$ is some effective external potential and

$$
\mathcal{B}_{\mathrm{s}}\left[n_{\mathrm{c}}\right]=\int_{z_{\mathrm{i}}}^{z_{\mathrm{f}}} \mathrm{d} z\left\langle\Phi_{\mathrm{c}, \mathrm{s}}\left[n_{\mathrm{c}}\right](z)\left|\mathrm{i} \frac{\partial}{\partial z}-\hat{T}\right| \Phi_{\mathrm{c}, \mathrm{s}}\left[n_{\mathrm{c}}\right](z)\right\rangle .
$$

The KS wave function is a Slater determinant of TD KS orbitals $\left\{\phi_{\mathrm{c}, i}(\mathbf{r} z)\right\}$ that satisfy:

$$
\mathrm{i} \frac{\partial \phi_{\mathrm{c}, i}}{\partial z}=\left(-\frac{1}{2} \nabla_{\mathbf{r}}^{2}+u_{\mathrm{c}, \mathrm{s}}\left[n_{\mathrm{c}}\right](\mathbf{r} z)\right) \phi_{\mathrm{c}, i}(\mathbf{r} z),
$$

where $u_{\mathrm{c}, \mathrm{s}}\left[n_{\mathrm{c}}\right]$ is the KS potential that represents $n_{\mathrm{c}}(\mathbf{r} z)$. Thus, if we differentiate $\mathcal{B}_{\mathrm{s}}$ with respect to the TD density we obtain:

$$
\frac{\delta \mathcal{B}_{\mathrm{s}}}{\delta n_{\mathrm{c}}(\mathbf{r} z)}=u_{\mathrm{c}, \mathrm{S}}\left[n_{\mathrm{c}}\right](\mathbf{r} z)+\int_{z}^{z_{\mathrm{f}}} \mathrm{d} z^{\prime} \int \mathrm{d}^{3} \mathbf{r}^{\prime} n_{\mathrm{c}}\left(\mathbf{r}^{\prime} z^{\prime}\right) \chi_{\mathrm{c}, \mathrm{s}}^{-1}\left[n_{\mathrm{c}}\right]\left(\mathbf{r}^{\prime} z^{\prime}, \mathbf{r} z\right),
$$

where $\chi_{\mathrm{c}, \mathrm{s}}^{-1}\left(\mathbf{r} z, \mathbf{r}^{\prime} z^{\prime}\right)=\delta u_{\mathrm{c}, \mathrm{s}}\left(\mathbf{r}^{\prime} z^{\prime}\right) / \delta n_{\mathrm{c}}(\mathbf{r} z)$.

Recall the Hartree functional:

$$
\mathcal{A}_{\mathrm{H}}\left[n_{\mathrm{c}}\right]=\frac{1}{2} \int_{z_{\mathrm{i}}}^{z_{\mathrm{f}}} \mathrm{d} z \int \mathrm{d}^{3} \mathbf{r} \int \mathrm{d}^{3} \mathbf{r}^{\prime} \frac{n_{\mathrm{c}}\left(\mathbf{r}^{\prime} z\right) n_{\mathrm{c}}(\mathbf{r} z)}{\left|\mathbf{r}-\mathbf{r}^{\prime}\right|} .
$$

Let us introduce the $\mathrm{XC}$ action functional:

$$
\mathcal{A}_{\mathrm{xc}}\left[n_{\mathrm{c}}\right]=\mathcal{B}_{\mathrm{s}}\left[n_{\mathrm{c}}\right]-\mathcal{B}\left[n_{\mathrm{c}}\right]-\mathcal{A}_{\mathrm{H}}\left[n_{\mathrm{c}}\right] .
$$

Using Eqs. (35) and (39) it is found that the functional derivative of the $\mathrm{XC}$ action functional can be expressed as:

$$
\begin{aligned}
u_{\mathrm{c}, \mathrm{xc}}(\mathbf{r} z)+\int_{z}^{z_{\mathrm{f}}} \mathrm{d} z^{\prime} \int \mathrm{d}^{3} \mathbf{r}^{\prime} n_{\mathrm{c}}\left(\mathbf{r}^{\prime} z^{\prime}\right)\left[\chi_{\mathrm{c}, \mathrm{s}}^{-1}\left(\mathbf{r}^{\prime} z^{\prime}, \mathbf{r} z\right)\right. \\
\left.-\chi_{\mathrm{c}}^{-1}\left(\mathbf{r}^{\prime} z^{\prime}, \mathbf{r} z\right)\right]=\frac{\delta \mathcal{A}_{\mathrm{xc}}}{\delta n_{\mathrm{c}}(\mathbf{r} z)}
\end{aligned}
$$

Here $u_{\mathrm{c}, \mathrm{xc}}(\mathbf{r} z)=u_{\mathrm{c}, \mathrm{s}}(\mathbf{r} z)-u_{\mathrm{c}}(\mathbf{r} z)-u_{\mathrm{c}, \mathrm{H}}(\mathbf{r} z)$, where the Hartree potential is $u_{\mathrm{c}, \mathrm{H}}\left[n_{\mathrm{c}}\right](\mathbf{r} z)=$ $\int \mathrm{d}^{3} \mathbf{r}^{\prime} n_{\mathrm{c}}\left(\mathbf{r}^{\prime} \mathbf{z}\right) /\left|\mathbf{r}-\mathbf{r}^{\prime}\right|$. Now introduce the XC kernel $f_{\mathrm{c}, \mathrm{xc}}\left(\mathbf{r} z, \mathbf{r}^{\prime} z^{\prime}\right)=\delta u_{\mathrm{c}, \mathrm{xc}}(\mathbf{r} z) / \delta n_{\mathrm{c}}\left(\mathbf{r}^{\prime} z^{\prime}\right)$, which satisfies:

$$
\chi_{\mathrm{c}, \mathrm{s}}^{-1}\left(\mathbf{r} z, \mathbf{r}^{\prime} z^{\prime}\right)=\chi_{\mathrm{c}}^{-1}\left(\mathbf{r} z, \mathbf{r}^{\prime} z^{\prime}\right)+\frac{\delta_{\mathrm{c}}\left(z-z^{\prime}\right)}{\left|\mathbf{r}-\mathbf{r}^{\prime}\right|}+f_{\mathrm{c}, \mathrm{xc}}\left(\mathbf{r} z, \mathbf{r}^{\prime} z^{\prime}\right) .
$$

The delta function in $\mathcal{C}$ space is defined such that $\int_{z_{\mathrm{i}}}^{z_{\mathrm{f}}} \mathrm{d} z^{\prime} f_{\mathrm{c}}\left(z^{\prime}\right) \delta_{\mathrm{c}}\left(z-z^{\prime}\right)=f_{\mathrm{c}}(z)$. The KS response function and the XC kernel satisfy the same properties of $\chi_{\mathrm{c}}$ regarding exchange of variables. 
In order to simplify Eq. (42), suppose that the density is physical, $n_{\mathrm{c}}=\bar{n}_{\mathrm{c}}$. This imposes that the XC potential is the same in both $\mathcal{C}^{+}$and $\mathcal{C}^{-}$spaces. For example, if $z=t^{+}$then the integral in time can be split up into two integrals: The first one runs from $t^{+}$to $t^{-}$, and the second one from $t^{-}$to $t_{0}^{-}$. There is no contribution from the first integral due to the symmetry properties of $\chi_{\mathrm{c}}^{-1}$ and $\chi_{\mathrm{c}, \mathrm{s}}^{-1}$ at physical densities. For the second integral we can use the antisymmetry relation to obtain in real-time that:

$$
u_{\mathrm{xc}}(\mathbf{r} t)+\int_{t_{0}}^{t} \mathrm{~d} t^{\prime} \int \mathrm{d}^{3} \mathbf{r}^{\prime} f_{\mathrm{xc}}\left(\mathbf{r} t, \mathbf{r}^{\prime} t^{\prime}\right) n\left(\mathbf{r}^{\prime} t^{\prime}\right)=\frac{\delta \mathcal{A}_{\mathrm{xc}}}{\delta n(\mathbf{r} t)},
$$

where $u_{\mathrm{xc}}(\mathbf{r} t)=\bar{u}_{\mathrm{c}, \mathrm{xc}}\left(\mathbf{r} t^{ \pm}\right)$and

$$
\frac{\delta \mathcal{A}_{\mathrm{xc}}}{\delta n(\mathbf{r} t)}=\left.\frac{\delta \mathcal{A}_{\mathrm{xc}}}{\delta n_{\mathrm{c}}\left(\mathbf{r} t^{ \pm}\right)}\right|_{n_{\mathrm{c}}=\bar{n}_{\mathrm{c}}} .
$$

Setting $z=t^{-}$in Eq. (42) also leads to Eq. (44) when $n_{\mathrm{c}}=\bar{n}_{\mathrm{c}}$; for this reason we expressed the final result in real-time. Because $f_{\mathrm{c}, \mathrm{xc}}$ in the $\mathcal{C}$ space also has the same properties as $\chi_{\mathrm{c}}^{-1}$ we identify the XC kernel in real-time, $f_{\mathrm{xc}}\left(\mathbf{r} t, \mathbf{r}^{\prime} t^{\prime}\right)$, as $\left.f_{\mathrm{c}, \mathrm{xc}}\left(\mathbf{r} t^{ \pm}, \mathbf{r}^{\prime} t^{\prime+}\right)\right|_{n_{\mathrm{c}}=\bar{n}_{\mathrm{c}}}$, or $-\left.f_{\mathrm{c}, \mathrm{xc}}\left(\mathbf{r}^{\prime} t^{\prime-}, \mathbf{r} t^{-}\right)\right|_{n_{\mathrm{c}}=\bar{n}_{\mathrm{c}}}$. Thus, the XC kernel is causal in real-time.

Given that we assumed that the response functions $\chi_{\mathrm{c}}$ and $\chi_{\mathrm{c}, \mathrm{s}}$ are strictly causal in $\mathcal{C}$, the integral in Eq. (42) is taken over the interval $\left(z, z_{\mathrm{f}}\right]$. This implies that the Hartree kernel $\delta_{\mathrm{c}}\left(z-z^{\prime}\right) /\left|\mathbf{r}-\mathbf{r}^{\prime}\right|$ lies outside the integration limits and thus it has no contribution to Eq. (44). Based on this, the integral in Eq. (44) is carried out strictly over the past of $t$, i.e., $\left[t_{0}, t\right)$. Hence, our causality assumption avoids singularities at equal-times and simplifies the transition to real-time.

Eq. (44) is the main result of this work. It is a variational equation that establishes a causal connection between $u_{\mathrm{xc}}$ in real-time with an XC action functional in the Keldysh space, and the memory of the system. If an approximation to the $\mathrm{XC}$ action functional is known, then Eq. (44) can be used to estimate the XC potential. The potentials $u(\mathbf{r} t)$ and $u_{\mathrm{s}}(\mathbf{r} t)$ also satisfy the same type of equation as that of $u_{\mathrm{xc}}$; one has to replace $f_{\mathrm{xc}}$ and $\mathcal{A}_{\mathrm{xc}}$ by $\chi^{-1}$ and $\mathcal{B}$, or $\chi_{\mathrm{s}}^{-1}$ and $\mathcal{B}_{\mathrm{s}}$.

Note that the left-hand side of Eq. (44) is a functional of the density $\bar{n}_{\mathrm{c}}\left(\mathbf{r} t^{ \pm}\right)$, or simply $n(\mathbf{r} t)$. This implies that the second functional derivative of $\mathcal{A}_{\mathrm{xc}}$ with respect to the density in real-time is not symmetric, i.e.:

$$
\frac{\delta}{\delta n\left(\mathbf{r}^{\prime} t^{\prime}\right)} \frac{\delta \mathcal{A}_{\mathrm{xc}}}{\delta n(\mathbf{r} t)}=0 \quad t^{\prime} \geq t .
$$

Here, the symbol $\delta / \delta n\left(\mathbf{r}^{\prime} t^{\prime}\right)$ represents regular functional differentiation in real-time because the operation $\delta / \delta n(\mathbf{r} t)$ already involves evaluation at the physical regime. The above result is a consequence of implementing causality in the $\mathcal{C}$ space explicitly using the path-ordering operator. Furthermore, recursive differentiation of Eq. (44) also allows us to express its solution as a series of functional derivatives of $\mathcal{A}_{\mathrm{xc}}$. This reads

$$
u_{\mathrm{xc}}\left(\mathbf{x}_{1}\right)=\frac{\delta \mathcal{A}_{\mathrm{xc}}}{\delta n\left(\mathbf{x}_{1}\right)}+w_{\mathrm{xc}}\left(\mathbf{x}_{1}\right)
$$


where

$$
w_{\mathrm{xc}}\left(\mathbf{x}_{1}\right)=\sum_{m=2}^{\infty} \frac{(-1)^{m+1}}{m !} \int \mathrm{d} \mu\left(\mathbf{x}_{2}\right) \cdots \mathrm{d} \mu\left(\mathbf{x}_{m}\right) \frac{\delta^{m-1}}{\delta n\left(\mathbf{x}_{m}\right) \cdots \delta n\left(\mathbf{x}_{2}\right)} \frac{\delta \mathcal{A}_{\mathrm{xc}}}{\delta n\left(\mathbf{x}_{1}\right)} .
$$

Here $\mathbf{x}_{m}=\mathbf{r}_{m}, t_{m}, m=1,2, \ldots$ and $\mathrm{d} \mu\left(\mathbf{x}_{m}\right)=n\left(\mathbf{x}_{m}\right) \mathrm{d}^{4} \mathbf{x}_{m}$. The functional derivatives in the integral are zero if, for any $i>j, t_{i} \geq t_{j}$. This series shows that the $\mathrm{XC}$ potential depends on perturbations of the $\mathrm{XC}$ potentials in all orders. However, in order to achieve convergence the functional derivatives must decrease as their order increases.

Now let us apply our variational equation to the derivation of the ALDA XC potential:

$$
\mathcal{A}_{\mathrm{xc}}^{\mathrm{ALDA}}\left[n_{\mathrm{c}}\right]=\left.\int_{z_{\mathrm{i}}}^{z_{\mathrm{f}}} \mathrm{d} z \int \mathrm{d}^{3} \mathbf{r}\left[\epsilon_{\mathrm{xc}}(n) n\right]\right|_{n=n_{\mathrm{c}}(\mathbf{r} z)},
$$

where $\epsilon_{\mathrm{xc}}$ is the local XC energy density. To solve Eq. (44) the memory term can be neglected to yield

$$
\bar{u}_{\mathrm{c}, \mathrm{xc}}^{\mathrm{ALDA}}(\mathbf{r} z)=\left.\frac{d}{d n}\left[\epsilon_{\mathrm{xc}}(n) n\right]\right|_{n=n_{\mathrm{c}}(\mathbf{r} z)} .
$$

Further differentiation leads to the kernel formula:

$$
f_{\mathrm{xc}}^{\mathrm{ALDA}}\left(\mathbf{r} t, \mathbf{r}^{\prime} t^{\prime}\right)=\left.\delta\left(\mathbf{r}-\mathbf{r}^{\prime}\right) \delta\left(t-t^{\prime}\right) \frac{d^{2}}{d n^{2}}\left[\epsilon_{\mathrm{xc}}(n) n\right]\right|_{n=n(\mathbf{r} t)} .
$$

The singularity of the XC kernel does not contribute to the integral term of Eq. (44) because the end point is not included, or in other words, the end point is approached in a limiting procedure. Hence, the above equation satisfies Eq. (44) and thus it is the solution of it. The singularity of the XC kernel arises from the definition of the XC potential, which implies that at equal-times the $\mathrm{XC}$ kernel must cancel the singularity of the Hartree kernel. However, the ALDA XC kernel does not cancel the singularity of the Hartree kernel due to the self-interaction error.

Another application is the TDOPM. The exchange functional form remains the same as the one proposed by van Leeuwen [25]:

$$
\mathcal{A}_{\mathrm{x}}\left[n_{\mathrm{c}}\right]=\int_{z_{\mathrm{i}}}^{z_{\mathrm{f}}} \mathrm{d} z\left\langle\Phi_{\mathrm{c}}\left[n_{\mathrm{c}}\right](z)|\hat{W}| \Phi_{\mathrm{c}}\left[n_{\mathrm{c}}\right](z)\right\rangle-\mathcal{A}_{\mathrm{H}}\left[n_{\mathrm{c}}\right]
$$

To derive the TDOPM one has to assume that (for example, see [3]):

$$
\frac{\delta \mathcal{A}_{\bar{v}_{\mathrm{c}}}}{\delta n(\mathbf{r} z)}=\frac{\delta \mathcal{A}_{\mathrm{s}, \bar{v}_{\mathrm{c}, \mathrm{s}}}}{\delta n(\mathbf{r} z)}
$$

If we set $\mathcal{A}_{\mathrm{xc}}=\mathcal{A}_{\mathrm{x}}$ and expand $A_{\bar{v}_{\mathrm{c}}}$ using Eq. (41) we find that the memory term in Eq. (44) can be discarded. Hence we can write:

$$
u_{\mathrm{x}}(\mathbf{r} t)=\frac{\delta \mathcal{A}_{\mathrm{x}}}{\delta n(\mathbf{r} t)} .
$$


The right hand side of the above equation can be calculated using the chain rule. If the result is multiplied by $\chi_{s}$ and then integrated, the final result coincides with that of Ullrich et al. [29].

Ground-state DFT is also accessible with this theory. We can introduce a slowly varying density $n_{\mathrm{c}}^{T}(\mathbf{r} z)=n_{\mathrm{c}}(\mathbf{r} z / T)$, where $T \rightarrow \infty$. One can use the adiabatic theorem to show that:

$$
\lim _{T \rightarrow \infty} A_{\text {xc }}\left[n_{\mathrm{c}}^{T}\right]=\lim _{T \rightarrow \infty} \int_{z_{\mathrm{i}}}^{z_{\mathrm{f}}} \mathrm{d} z E_{\mathrm{xc}}\left[n_{\mathrm{c}}^{T}(\cdot, z)\right],
$$

where $E_{\mathrm{xc}}$ is the XC energy functional of DFT. The above equation is local in time. As in the previous case, the solution of Eq. (44) has to be of the form:

$$
\lim _{T \rightarrow \infty} u_{\mathrm{xc}}\left[n^{T}\right](\mathbf{r} t)=\left.\lim _{T \rightarrow \infty} \frac{\delta E_{\mathrm{xc}}}{\delta n(\mathbf{r})}\right|_{n=n^{T}(\mathbf{r} t)} .
$$

where $n^{T}=\bar{n}_{\mathrm{c}}^{T}$.

\section{Conclusions}

To summarize, we examined the RG action functional and the solution of the causality paradox by Vignale [26] from the point of view of unitary evolution. We extended this solution to the Keldysh space, and, under the strict causality assumption, we found a variational equation for the XC potential that involves an XC memory term. The solution of this variational equation is a series in terms of functional derivatives of the $\mathrm{XC}$ action functional in the Keldysh space. We showed that it is possible to derive the ALDA XC and TDOPM exchange potentials from the present theory and that ground states are also accessible using the adiabatic theorem.

\section{Acknowledgements}

The author thankfully acknowledges valuable discussion with Adam Wasserman, Daniel Jensen, and Daniel Whitenack.

\section{References}

[1] E. Gross and W. Kohn. Time-Dependent Density-Functional Theory, volume 21 of Adv. Quantum Chem., pages 255-291. Elsevier, 1990.

[2] M. A. L. Marques and E. K. U. Gross. Time-dependent density-functional theory. Annu. Rev. Phys. Chem., 55(1):427-455, 2004.

[3] Carsten A. Ullrich. Time-Dependent Density-Functional Theory: Concepts and Applications (Oxford Graduate Texts). Oxford University Press, USA, February 2012. 
[4] Kieron Burke, Jan Werschnik, and E. K. U. Gross. Time-dependent density functional theory: Past, present, and future. J. Chem. Phys., 123(6):062206+, 2005.

[5] M. Lein and E. K. U. Gross. Back to the ground-state: Electron gas. In Miguel A.L. Marques, Carsten A. Ullrich, Fernando Nogueira, Angel Rubio, Kieron Burke, and E. K. U. Gross, editors, Time-Dependent Density Functional Theory, volume 706 of Lecture Notes in Physics, page 423. Springer Berlin Heidelberg, 2006.

[6] Andreas Hesselmann and Andreas Görling. Correct Description of the Bond Dissociation Limit without Breaking Spin Symmetry by a Random-Phase-Approximation Correlation Functional. Phys. Rev. Lett., 106:093001+, February 2011.

[7] Erich Runge and E. K. U. Gross. Density-Functional Theory for Time-Dependent Systems. Phys. Rev. Lett., 52(12):997+, March 1984.

[8] Robert van Leeuwen. Mapping from Densities to Potentials in Time-Dependent DensityFunctional Theory. Phys. Rev. Lett., 82:3863-3866, May 1999.

[9] D.J. Tozer. Relationship between long-range charge-transfer excitation energy error and integer discontinuity in kohn-sham theory. J. Chem. Phys., 119:12697, 2003.

[10] M. Hellgren and EKU Gross. Discontinuities of the exchange-correlation kernel and charge-transfer excitations in time-dependent density-functional theory. Phys. Rev. A, 85(2):022514, 2012.

[11] S. Kurth, G. Stefanucci, E. Khosravi, C. Verdozzi, and EKU Gross. Dynamical coulomb blockade and the derivative discontinuity of time-dependent density functional theory. Phys. Rev. Lett., 104(23):236801, 2010.

[12] M. Koentopp, C. Chang, K. Burke, and R. Car. Density functional calculations of nanoscale conductance. J. Phys.-Condens. Mat., 20(8):083203, 2008.

[13] K. Burke, R. Car, and R. Gebauer. Density functional theory of the electrical conductivity of molecular devices. Phys. Rev. Lett., 94(14):146803, 2005.

[14] Jeffrey L. Krause, Kenneth J. Schafer, and Kenneth C. Kulander. Phys. Rev. Lett, 68: 3535-3538, 1992.

[15] N.T. Maitra, F. Zhang, R.J. Cave, and K. Burke. Double excitations within timedependent density functional theory linear response. J. Chem. Phys., 120:5932, 2004.

[16] P. Elliott, F. Furche, and K. Burke. Excited states from time-dependent density functional theory. Rev. Comp. Ch., 26:91-165, 2009.

[17] M. Ruggenthaler and R. van Leeuwen. Global fixed-point proof of time-dependent density-functional theory. Europhys. Lett., pages 13001+, July 2011. 
[18] M. Ruggenthaler, KJH Giesbertz, M. Penz, and R. van Leeuwen. Density-potential mappings in quantum dynamics. Phys. Rev. A., 85(5):052504, 2012.

[19] Roi Baer. On the mapping of time-dependent densities onto potentials in quantum mechanics. J. Chem. Phys., 128(4):044103+, 2008.

[20] Søren Ersbak Bang Nielsen, Micheal Ruggenthaler, and Robert van Leeuwen. Manybody quantum dynamics from the density. Europhys. Lett., 101(3):33001, 2013.

[21] V. Peuckert. J. Phys. C Solid State, 11(24):4945, February 2001.

[22] E.K.U. Gross, J.F. Dobson, and M. Petersilka. Density functional theory of timedependent phenomena. In R.F. Nalewajski, editor, Density Functional Theory II, volume 181 of Topics in Current Chemistry, pages 81-172. Springer Berlin Heidelberg, 1996.

[23] A. K. Rajagopal. Time-dependent variational principle and the effective action in density-functional theory and Berry's phase. Phys. Rev. A, 54:3916-3922, November 1996.

[24] R. Jackiw and A. Kerman. Phys. Lett. A, 71(2-3):158, April 1979.

[25] Robert van Leeuwen. Causality and Symmetry in Time-Dependent Density-Functional Theory. Phys. Rev. Lett., 80(6):1280-1283, February 1998.

[26] Giovanni Vignale. Real-time resolution of the causality paradox of time-dependent density-functional theory. Phys. Rev. A, 77:062511+, June 2008.

[27] Michael Ruggenthaler and Robert Leeuwen. Beyond the runge-gross theorem. In Miguel A.L. Marques, Neepa T. Maitra, Fernando M.S. Nogueira, E.K.U. Gross, and Angel Rubio, editors, Fundamentals of Time-Dependent Density Functional Theory, volume 837 of Lecture Notes in Physics, pages 187-210. Springer Berlin Heidelberg, 2012.

[28] J. Daligault. Variational formulation of time-dependent density functional theory. arXiv:1210.6938, 2012.

[29] C. A. Ullrich, U. J. Gossmann, and E. K. U. Gross. Time-Dependent Optimized Effective Potential. Phys. Rev. Lett., 74(6):872-875, February 1995. 